\title{
Monte Carlo simulations in zeolites
}

\author{
Berend Smit*, R. Krishna \\ Department of Chemical Engineering, Universiteit van Amsterdam, Nieuwe Achtergracht 166, 1018 WV Amsterdam, The Netherlands
}

\begin{abstract}
In this short review, the applications of Monte Carlo simulations to the study of the adsorption and diffusion of hydrocarbons in zeolites are discussed. We focus on those systems for which the conventional molecular simulation techniques, molecular dynamics and Monte Carlo, are not sufficiently efficient. In particular, to simulate the adsorption and diffusion of long-chain hydrocarbons, novel Monte Carlo techniques have been developed. Here we discuss configurational-bias Monte Carlo (CBMC) and kinetic Monte Carlo (KMC). CBMC was developed to compute the thermodynamic properties. KMC is applied to compute transport properties. The use of these methods is illustrated with examples of technological importance. (C) 2001 Elsevier Science Ltd. All rights reserved.
\end{abstract}

Keywords: Zeolites; Adsorption; Diffusion; Monte Carlo simulations; Kinetic Monte Carlo

\section{Introduction}

Zeolites are microporous materials that can form a one-, two-, or three-dimensional framework. These types of materials are used as catalysts in many petrochemical processes. A typical catalytic application is dewaxing, in which long-chain alkanes (waxes) are converted into the more valuable branched isomers. Other applications involve separation, using, for example, a zeolite membrane as a molecular sieve. The behaviour of molecules adsorbed in the pores of a zeolite is difficult to study experimentally. Most experiments yield important macroscopic data, for example heats of adsorption or adsorption isotherms, from which one can only indirectly extract molecular information on these adsorbed molecules. Therefore, molecular simulation is an attractive alternative to obtain such information.

In this short review we discuss recent developments in the use of Monte Carlo schemes to study adsorption and diffusion in zeolites. We focus on configurational-bias Monte Carlo (CBMC) and kinetic Monte Carlo (KMC). As we will show in the next section the conventional simulation techniques (molecular dynamics and Monte Carlo) are not sufficiently efficient to simulate long-chain hydrocarbons. The CBMC technique was developed to compute the thermodynamic properties of chain molecules efficiently, while kinetic Monte Carlo is used to study the

\footnotetext{
*Corresponding author.

E-mail addresses: smit@its.chem.uva.nl (B. Smit), krisna@its.chem.uva.nl (R. Krishna).
}

diffusion of these molecules. Finally, we discuss some applications of these techniques in the context of the adsorption and diffusion of molecules adsorbed in zeolites. In particular, we discuss some applications in which such simulations have helped us to obtain a better understanding of these materials.

\section{Conventional molecular simulations}

Several molecular simulation techniques have been used to study adsorption in zeolites. The earlier studies used Molecular Mechanics to study the conformation or docking of molecules. From a computational point of view such simulations are relatively simple since they only involve the conformation of the molecule with the lowest energy. From a Statistical Thermodynamics point of view such a conformation corresponds to the equilibrium distribution at $T=0 \mathrm{~K}$, where entropy effects do not play a role. All applications of zeolites, however, are at elevated temperatures. Simulations under these conditions require the use of molecular dynamics or Monte Carlo techniques. For such simulations, one needs to sample many millions of configurations, which requires much more CPU time.

Because of the CPU requirement, most of the systems that have been studied by Monte Carlo techniques and molecular dynamics concern the adsorption of noble gases or methane. Only a few studies of ethane or propane have been published. Only very recently have computers become sufficiently powerful to perform molecular dynamics simulations of long-chain alkanes [1,2]. The reason why 
only small molecules have been studied becomes clear from the work of June et al. [3], in which molecular dynamics was used to investigate the diffusion of butane and hexane in the zeolite silicalite. June et al. showed that the diffusion of butane from one channel of the zeolite into another channel is very slow compared with the diffusion of bulk butane. As a consequence, many hours of supercomputer time were required to obtain reliable results. In addition, diffusion decreases significantly with increasing chain length.

The above example illustrates the fundamental problem of molecular dynamics. In a molecular dynamics simulation the approach is to mimic the behaviour of the molecules as good as possible. If successful, all properties will be as in nature, including diffusion. If the molecules diffuse slowly, this will be reflected in very long simulation times and, in the case of long-chain alkanes, these simulation times have only recently be attained.

In principle, one can circumvent this intrinsically slow dynamics by using a Monte Carlo technique. In a Monte Carlo simulation, one does not have to follow the "natural path' and one can, for example, perform a move in which it is attempted to displace a molecule to a random position in the zeolite. If such a move is accepted, it corresponds to a very large jump in phase space. Again, the utilisation of such 'un-natural' Monte Carlo moves turned out to be limited to small molecules. For example, Goodbody et al. [4] used this Monte Carlo trick to determine the adsorption isotherm of methane in silicalite. In such a simulation, one can observe that, out of 1000 attempts to move a methane molecule to a random position in the zeolite, 999 attempts will be rejected because the methane molecule overlaps with a zeolite atom. If we were to perform a similar move with an ethane molecule, we would need $1000 \times 1000$ attempts to have one that was successful. Clearly, this random insertion scheme will break down for any but the smallest alkanes.

\section{Monte Carlo simulation of chain molecules}

It may not be obvious why we need efficient Monte Carlo methods to simulate chain molecules. In general, a molecular dynamics approach is much easier to generalise to complex molecules. An example of an experiment that is 'impossible' to simulate using molecular dynamics is the computation of an adsorption isotherm. One needs a reservoir that is in open contact with a zeolite. For longchain hydrocarbons, equilibration in the laboratory may take hours or even several days. It would be very impractical to mimic this experiment with a simulation. Even if one would have the patience to wait several million years before our computer experiment is equilibrated, one has to worry about the zeolite surface and one has to simulate a large reservoir of uninteresting molecules. It is, therefore, much more convenient to perform a grand-canonical
Monte Carlo simulation. In such a simulation, one imposes the temperature and chemical potential and computes the average number of particles in the (periodically) repeated zeolite crystal. It is important to note that, in such a simulation, the number of particles is not fixed but varies during the simulation. In such a simulation, one therefore has to perform Monte Carlo moves which attempt to add or to remove particles. To apply such a simulation for a long-chain alkane, one has to be able to insert such a molecule into a zeolite. In the Introduction we have seen that such moves are impossible to perform by a conventional Monte Carlo simulation technique.

To make Monte Carlo simulations of long-chain molecules possible the configurational-bias Monte Carlo (CBMC) technique was developed [**5]. The principal idea of the configurational-bias Monte Carlo technique is to grow a molecule atom by atom instead of attempting to insert the entire molecule at random. Fig. 1 shows one of the steps in this algorithm. It is important to note that this growth procedure introduces a bias, such that only the most favourable configurations are generated. If one were to use the ordinary Metropolis acceptance rule, such a bias in the configurations of the molecules would lead to an incorrect distribution of configurations. This bias can be removed exactly by adjusting the acceptance rules [**5].

It is not the purpose of this review to give an extensive discussion on the implementation of this algorithm for the adsorption of linear and branched alkanes in zeolites; details can be found in Refs. [6,**7]. Smit and Siepmann estimated that, for the adsorption of dodecane in silicalite, a CBMC simulation can be up to 10-20 orders of magnitude (!) more efficient than conventional techniques [8]. In the next section, we demonstrate that this enormous increase in efficiency allows us to perform simulations that were considered impossible only 10 years ago.

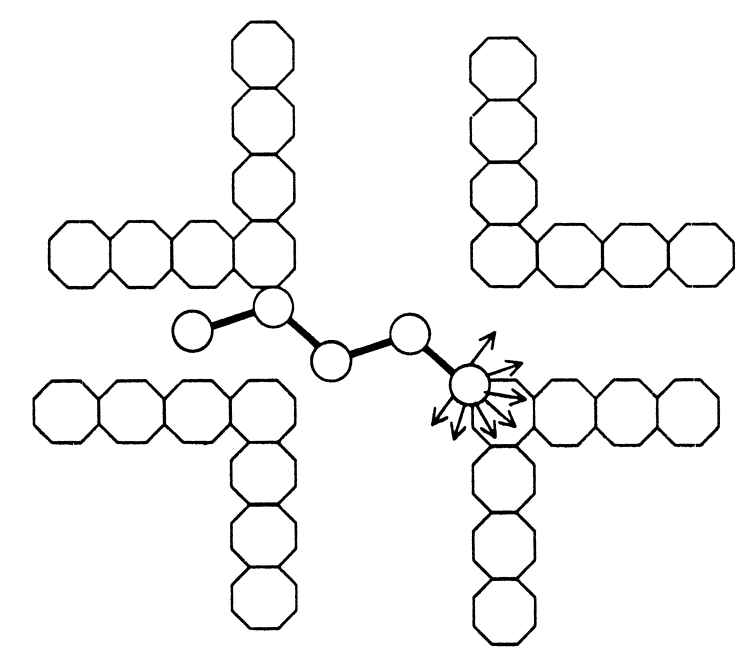

Fig. 1. Schematic drawing of the growth of an alkane in a zeolite in a CBMC move. The octagons represent the atoms of the zeolite and the circles represent the atoms of the alkane. Four atoms have been inserted successfully, and an attempt is made to insert the fifth. 
Most simulations have been performed on rigid zeolites, i.e. the atoms of the zeolite do not move during a simulation. This assumption reduces the CPU time significantly. It seems that for static properties, heats of adsorption, Henry coefficients, or adsorption isotherms, this may be a reasonable assumption. Most results reported for rigid zeolite lattices do not give a clear indication that this assumption may not hold. However, it is well known that adsorbents may induce phase transitions in the structure of the zeolite. Therefore, this clearly indicates that, eventually, one has to use a fully flexible zeolite. In addition, for dynamic properties, such as a diffusion coefficient, this assumption may lead to serious errors. The diffusion of bulky molecules (branched alkanes) may involve hopping over free energy barriers. The height of such a free energy barrier may be significantly lower if a zeolite is flexible.

The adsorbed molecules are often described with a united atom model. For example, in a hydrocarbon the $\mathrm{CH}$, $\mathrm{CH}_{2}$, and $\mathrm{CH}_{3}$ groups are considered as single united atoms. Despite the simplification these models do very well in reproducing the thermodynamic data of liquid hydrocarbons [9]. Also, one has to keep in mind that such a model has its limitations. For example, it is well known that a united atom model of an alkane cannot reproduce the experimental crystal structure. Details on the parameters of the various models can be found in Ref. [**7].

\section{Kinetic Monte Carlo}

In the previous section we focussed on the thermodynamic properties of chain molecules adsorbed in zeolites. For many applications, insight into the dynamic properties is also important, for example particularly in catalytic reactions and separation processes [**10]. A variety of models and techniques have been used to describe diffusion within zeolites, ranging from phenomenological models, such as Fick's law of diffusion, irreversible thermodynamics and the Maxwell-Stefan formulation, to Kinetic Monte Carlo simulations and Molecular Dynamics [**10,**11,12,13]. Additionally, transition path sampling and infrequent event simulation techniques have been used for estimation of the zeroloading diffusivities [14-16].

Different diffusion coefficients are defined for different applications [**17]. For the design of separation and reaction equipment, the transport, or Fick, diffusivity is required. The Fick diffusivity $D$ is measured under nonequilibrium conditions in which finite gradients of molecular loading exist. They are determined by macroscopic methods such as gravimetry, volumetry, chromatography or frequency response techniques [**10,18]. The transport diffusivity $D$ is strongly influenced by the sorption behaviour and it is common in practice to calculate the corrected, or jump, diffusivity. The jump diffusivity is

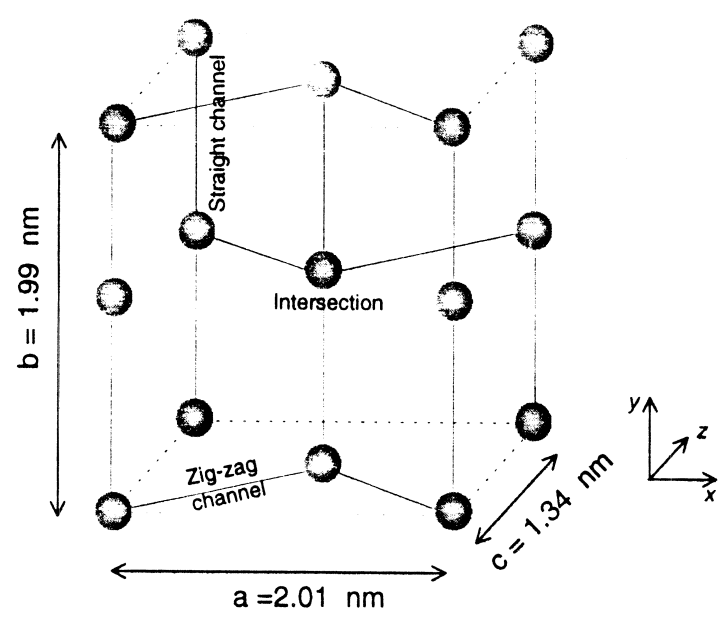

Fig. 2. Schematic structure of the silicalite-1 zeolite; the lines represent the zeolite channels and the spheres the intersections of linear and zig-zag channels.

more easily related to the activated jump processes of molecules. In other experimental procedures, the selfdiffusivities $D^{*}$ are measured under equilibrium conditions by microscopic techniques, viz. quasielastic neutron scattering and pulsed field gradient NMR [**10,18].

In this review we focus on the applications of Monte Carlo methods. In particular, we discuss the insights provided by KMC simulations in describing zeolite diffusion. For the applications of molecular dynamics we refer to Ref. [13]. A KMC simulation is not based on a molecular model (force field), but on a course-grained description. The pore space through which molecules diffuse is simplified to a lattice, consisting of a grid of sorption sites, which are connected by bonds. As an example we show the KMC setup for silicalite-1 in Fig. 2, where the large black spheres represent the intersection sites. Molecules occupy a certain fraction $\theta$ of the sites, and are assumed to hop from site to site, normally along bonds connecting neighboring sites. A sufficiently large number of molecular trajectories is followed, and statistics for quantities of interest are collected after the system has equilibrated. Diffusivities and other properties are then calculated based on ensemble averaged statistics. Since this is a coursed-grained description there is no unique recipe of how to map a zeolite onto a lattice model. Depending on the system and the properties of interest, other mappings of silicalite-1 can be introduced.

\section{Applications}

In this section we review some of the applications of Monte Carlo simulations in zeolites. At this point we would like to emphasise that we have made a small selection of the available literature and many important applications have been left out. We selected a few applica- 
tions that could not have been carried out without the use of modern Monte Carlo simulations.

\subsection{Adsorption isotherms of pure components}

In the Introduction we indicated that an important advantage of the Monte Carlo technique is the possibility to perform simulations in the Grand-canonical ensemble. This ensemble allows us to simulate an adsorption isotherm efficiently. Fig. 3 shows a comparison of simulated and experimental adsorption isotherms of linear and branched alkanes in the zeolite silicalite. The agreement is surprisingly good; the simulations reproduce all the qualitative features found in the experiments.

The simulations made it possible to give a molecular explanation of the steps in the adsorption isotherms. For example, Smit and Maesen [19] relate the plateau in the adsorption isotherm of heptane to a commensurate freezing of the hydrocarbons induced by the zig-zag channels of the zeolite. The plateau of the branched alkanes was shown to be related to the preference of these molecules for the intersections of the zig-zag and straight channels of this zeolite [**7]. If all intersections are occupied, an additional molecule has to reside on a less favourable position, and hence requires a higher pressure.

The good agreement of the simulated adsorption isotherms with the experimental ones is an encouraging result since experimental adsorption isotherms are not readily available for a given zeolite under a given condition. These results show that one can obtain a reasonable estimate from a molecular simulation. However, it is important to point out that most simulations have been performed for silicalite, for which the potentials have also been developed. Unfortunately, there are not many experimental adsorption isotherms of other all-silica zeolites. It is therefore not known how accurately these simulations extrapolate to other zeolites.

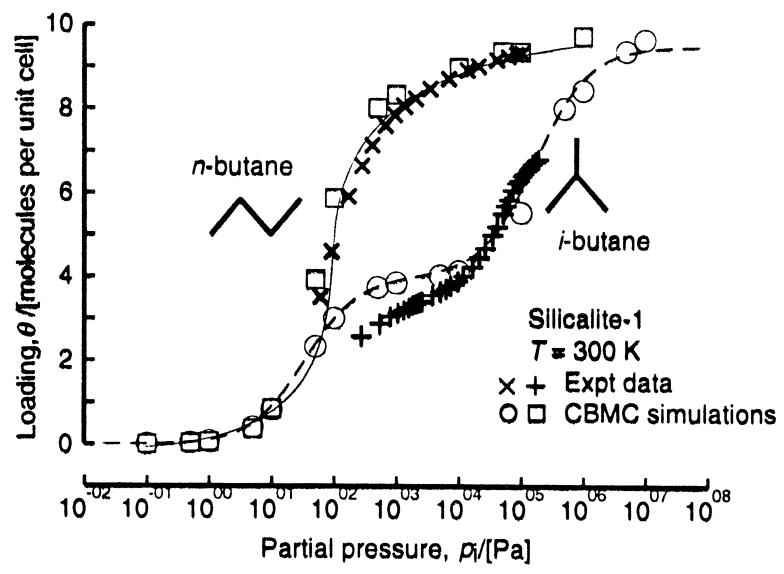

Fig. 3. Sorption isotherms for normal and iso-butane at $300 \mathrm{~K}$. Comparison of CBMC simulation with experiment [27].

\subsection{Separations of hydrocarbons using zeolite membranes}

At first sight it may not be very obvious how Monte Carlo simulation can contribute to the understanding of permeation through membranes. A description of permeation through membranes requires knowledge of the fluxes as a function of the concentration of the molecules. Krishna and Wesselingh [**17] have shown that the fluxes can be described using the Maxwell-Stefan equation, which relates the fluxes to the diffusion coefficient and the gradient of the chemical potential. This relation tells us that we need to know the chemical potential as a function of the loading of the molecules in the zeolite. To be more precise, one needs to know the loading of each individual component. For pure components there are not many experimental adsorption isotherms, and for mixtures it is even worse. There are simply many more mixtures one has to measure at various compositions, compared to pure components. Also, the conventional way to measure an adsorption isotherm is to monitor the weight increase of the sample as a function of the pressure. This is not sufficient for a mixture, and one has to perform an additional experiment to measure the composition of the adsorbed molecules. Therefore, most estimations of permeation are based on experimental Henry coefficients of the pure components.

Fig. 4 shows pure component isotherms and a mixture isotherm for a mixture of linear and branched isomers. At low pressures the adsorption isotherm is simply the sum of the pure component isotherms. At elevated pressures,

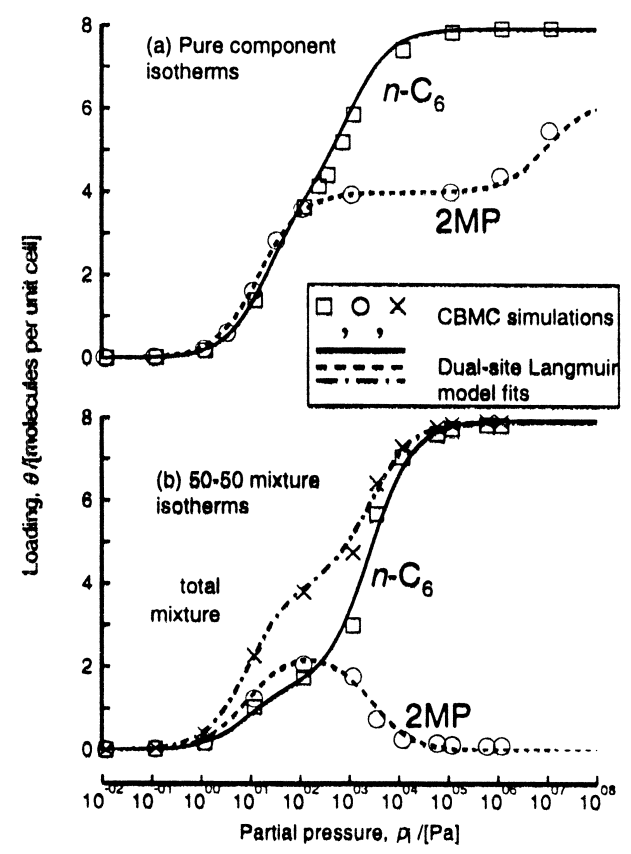

Fig. 4. Configurational-bias Monte Carlo simulations of the adsorption isotherms of n-hexane and 2-methyl pentane at $T=300 \mathrm{~K}$ [**7]. 
however, one observes that the branched alkane is expelled from the zeolite. For permeation through a membrane of a mixture of these components, these results indicate an enhancement of the selectivity by a factor of 20 compared to what one can predict on the basis of the pure components. In fact, these observations indicate that such mixture effects can be used for a novel concept to separate mixtures of hydrocarbons [20].

\subsection{Structure of zeolites}

An exciting application of advanced Monte Carlo methods was developed by Falcioni and Deem [**21]. The interesting aspect is that their method, from a technical point of view, is similar to the methods discussed in this review. However, Falcioni and Deem did not consider a molecular model of a zeolite. Their application involves determining the crystal structure of a zeolite when there is insufficient experimental data to use conventional techniques. There are about 118 different known zeolite structures. But many more zeolite structures are theoretically possible. If one makes a new zeolite, one often has a powder and it is much more time consuming, if possible at all, to make a sufficiently large crystal from which the crystal structure can be determined. Falcioni and Deem developed a Monte Carlo procedure to solve the structure of the zeolite [**21]. The problem is to find the crystal structure that corresponds best to an experimental powder diffraction pattern. Experimentally, it well known that, in zeolites, the angles between three successive T-sites (positions of the $\mathrm{Si}$ atoms in an all-silica zeolite) are clustered around $109.5^{\circ}$. Falcioni and Deem assumed that any new zeolite structure should have angles with a similar distribution. In a similar way, rules were deduced for the average bond distance and other properties of the structures. These rules were translated into a pseudo-Hamiltonian; this Hamiltonian has high values for those crystal structures that do not resemble the characteristics of a zeolite and a low value for crystals that have zeolite-like characteristics. An additional term was added to this structural pseudo-Hamiltonian that indicated the difference between the experimental and the calculated X-ray powder pattern of the new crystal structure. Falcioni and Deem then assumed that the unknown zeolite structure corresponds to the structure that minimises this combined Hamiltonian. Various Monte Carlo rules were invented to change the crystal structure and a new structure was accepted or rejected using this pseudo-Hamiltonian, or effective force field. Since this pseudo-Hamiltonian has many local minima, the so-called advanced Monte Carlo methods were used to find the optimal structure. To test their method, Falcioni and Deem used the information of 32 zeolite structures to generate the pseudo-Hamiltonian. This information was sufficient to solve the structure of all remaining known zeolites.

\subsection{Transport, jump and self-diffusivities}

The KMC simulations allows us to compare the transport $(D)$, jump $(D)$ and self-diffusivities $\left(D^{*}\right)$ for 2 methyl-hexane in silicalite-1. The results of Paschek and Krishna [22] are shown in Fig. 5. We first note that the jump and self-diffusivities decrease with increasing loading. Indeed, KMC simulations provide a convenient way to determine the loading dependence of the diffusivities for various topologies. For $2 \mathrm{MH}$ in silicalite, the jump diffusivity $D$ shows a linear dependence on the fractional vacancy $(1-\theta)$ and the transport, $D$, is seen to be independent of occupancy. It is a characteristic of KMC simulations that the diffusivities reduce to zero when the zeolite is totally occupied. In practice, diffusivities do not reduce to zero because other phenomena such as collective diffusion play a role; these cannot be handled within the KMC framework. From Fig. 5 we note that the selfdiffusivity $D^{*}$ values are lower than those for jump diffusivity; this difference is entirely due to the correlation effects associated with molecular jumps and can be modelled within the Maxwell-Stefan description with the aid of exchange coefficients [22]. Such correlation effects can be expected to depend on the zeolite topology, connectivity and the number of sorption sites occupied by the molecule, and KMC simulations provide a means of studying and quantifying such effects.

In the KMC simulations shown in Fig. 5, moleculemolecule interactions (attractive or repulsive forces) are not taken into account; such interactions can also be incorporated into the KMC methodology [23]. With increased molecular loading, repulsive interactions have the effect of enhancing the diffusivity and could provide an explanation for the many types of experimentally observed dependences of self-diffusivity on loading [24].

Correlated jumps of molecules have a more pronounced effect on mixture diffusion; they tend to speed up the slower molecule and slow down the molecule with a

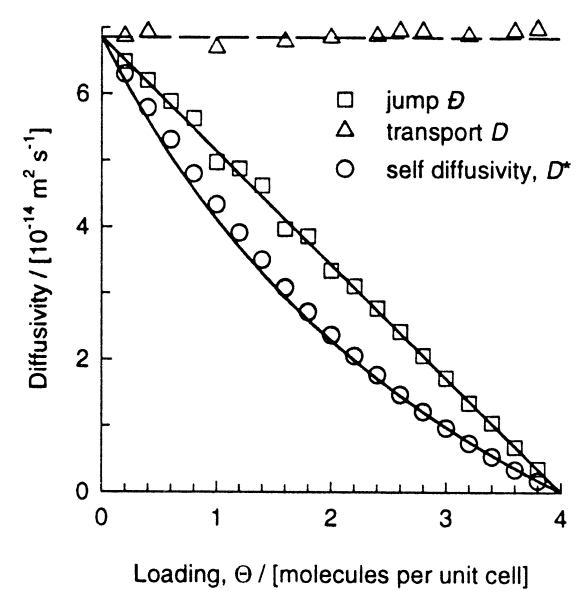

Fig. 5. KMC simulations of jump $(D)$, transport $(D)$, and self-diffusivities $\left(D^{*}\right)$ of $2 \mathrm{MH}$ in silicalite at $300 \mathrm{~K}$ [22]. 


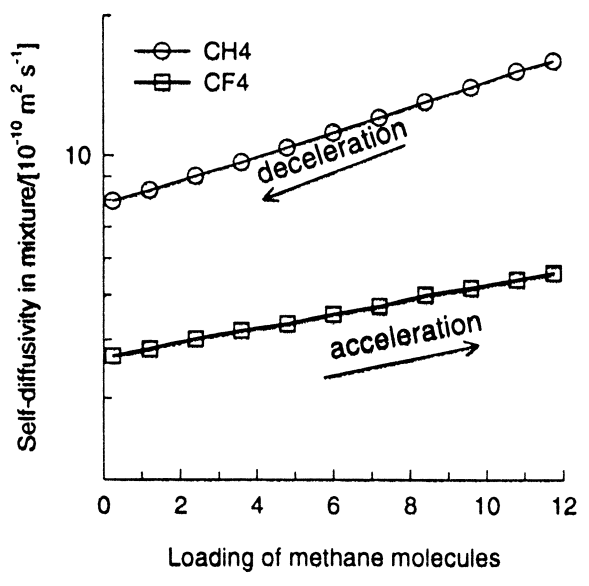

Fig. 6. KMC simulations of self-diffusivities for $\mathrm{CH}_{4}$ and $\mathrm{CF}_{4}$ in silicalite-1 at $200 \mathrm{~K}$ in a binary mixture with a total loading of 12 molecules per unit cell.

higher mobility. KMC simulations [25] for a mixture of $\mathrm{CH}_{4}$ and $\mathrm{CF}_{4}$ at $200 \mathrm{~K}$ and a total loading of 12 molecules per unit cell in silicalite are shown in Fig. 6. An increase in the fraction of the slower moving $\mathrm{CF}_{4}$ in the mixture tends to decelerate $\mathrm{CH}_{4}$, while an increase in the fraction of the faster moving $\mathrm{CH}_{4}$ tends to accelerate $\mathrm{CF}_{4}$; these results are also confirmed by MD simulations and experiment [*26]. KMC simulations provide a means of studying the acceleration-deceleration effects of various mixtures and varying topologies. Also, they provide a convenient means of validating mean-field continuum models for mixture diffusion such as the Maxwell-Stefan theory [25]. However, an important limitation of the KMC methodology as applied to mixtures needs to be underlined. The number of sorption sites which either molecule can occupy is necessarily the same in a KMC simulation. In practice, for mixtures of molecules of different size, e.g. methane and propane, there will be a significant difference in the saturation loadings of the constituents; such differences will influence the mixture diffusion behaviour. In the current implementations of KMC techniques, differences in maximum sorption loadings cannot be taken into account [25].

\section{Concluding remarks}

In this short review we have discussed the use of modern Monte Carlo simulations in the context of simulating the properties of molecules adsorbed in zeolites and solving the structure of the zeolites.

We have shown that it is possible to use Monte Carlo simulations to obtain a reasonable estimate of an adsorption isotherm of pure hydrocarbons and their mixtures in zeolites. These adsorption isotherms play an important role in understanding the various applications of zeolites. Most of these simulations have been on ideal all-silica zeolites that are perfect crystals. In practice, zeolites are never perfect and hardly ever contain silica only. Such an effect can, in principle, be taken into account in a molecular simulation, but it also requires experimental data on systems that have been carefully characterized.

Because of its course-grained character, kinetic Monte Carlo is an appropriate technique to study transport in zeolites for those system for which the diffusion is too slow to be studied using conventional molecular dynamics. An important prerequisite is that microscopic information on the hopping rates should be available. In most cases, experimental data will not be available and one has to use molecular simulations. In particular, the combination of rare event simulations with kinetic Monte Carlo appears to be a very promising route.

\section{Acknowledgements}

The authors gratefully acknowledge grants from the Netherlands Organisation for Scientific Research (NWOCW).

\section{References}

Papers of particular interest, published within the annual period of review, have been highlighted as:

* of special interest;

** of outstanding interest.

[1] Runnebaum RC, Maginn EJ. J Phys Chem B 1997;101:6394.

[2] Webb III EB, Grest GS, Mondello M. J Phys Chem B 1999; 103:4949.

[3] June RL, Bell AT, Theodorou DN. J Phys Chem 1992;96:1051.

[4] Goodbody SJ, Watanabe K, MacGowan D, Walton JPRB, Quirke N. J Chem Soc, Faraday Trans 1991;87:1951.

[**5] Frenkel D, Smit B. Understanding molecular simulations: from algorithms to applications, San Diego: Academic Press, 1996, a book for anyone who wants to understand simulations.

[6] Vlugt TJH, Martin MG, Smit B, Siepmann JI, Krishna R. Mol Phys 1998;94:727.

[**7] Vlugt TJH, Krishna R, Smit B. J Phys Chem B 1999;103:1102, "Comprehensive development of CBMC strategy to determine pure component and mixture isotherms for linear and branched alkanes in silicalite; extensive comparisons with experiments validate the strategy."

[8] Smit B, Siepmann JI. Science 1994;264:1118.

[9] Smit B, Karaborni S, Siepmann JI. J Chem Phys 1995;102:2126, erratum: J Chem Phys 1998;109:352.

[**10] Kärger J, Ruthven DM. Diffusion in zeolites and other microporous solids, New York: Wiley, 1992, this is the standard book for zeolite diffusion and contains a thorough discussion of all published experimental data and theoretical models.

[**11] Auerbach SM. Int Rev Phys Chem 2000;19:155, excellent up-todate review on modelling strategies for diffusion in zeolite.

[12] Coppens MO, Bell AT, Chakraborty AK. Chem Eng Sci 1998;53:2053.

[13] Demontis P, Suffritti GB. Chem Rev 1997;97:2845.

[14] Pal S, Fichthorn KA. Chem Eng J 1999;74:77. 
[15] Smit B, Loyens LDJC, Verbist GLMM. Faraday Discuss 1997;106:93.

[16] Vlugt TJH, Dellago C, Smit B. J Chem Phys 2000;113:8791.

[**17] Krishna R, Wesselingh JA. Chem Eng Sci 1997;52:861, review on the application of the Maxwell-Stefan approach in chemical engineering applications.

[18] Jobic H, Kärger J, Bée M. Phys Rev Lett 1999;82:4260.

[19] Smit B, Maesen TLM. Nature 1995;374:42.

[20] Krishna R, Smit B. Chem Innov 2001;31:27.
[**21] Falcioni M, Deem MW. J Chem Phys 1999;110:1754, very original application of advanced Monte Carlo methods.

[22] Paschek D, Krishna R. Phys Chem Chem Phys 2000;2:2389.

[23] Bhide SY, Yashonath S. J Phys Chem B 2000;104:2607.

[24] Kärger J, Pfeifer H. Zeolites 1987;7:90.

[25] Paschek D, Krishna R. Langmuir 2001;17:247.

[*26] Snurr RQ, Kärger J. J Phys Chem B 1997;101:6469.

[27] Vlugt TJH, Zhu W, Kapteijn F, Moulijn JA, Smit B, Krishna R. J Am Chem Soc 1998;120:5599. 